\title{
Processo de implantação e estruturação do Núcleo de Inovação Tecnológica
}

Iana Suly Santos Katz

Mestre e Doutora em Ciências pela Universidade de São Paulo (USP). Fez MBA em Gestão da Inovação em Saúde no Instituto Butantan. Participa do Programa de Desenvolvimento em Gestão da Fundação Dom Cabral. Atualmente é Coordenadora do Núcleo de Inovação tecnológica e membro da Comissão Científica do Instituto Pasteur.

ianasuly@gmail.com

Flávia Oliveira do Prado

Doutora em Administração de Organizações pela Universidade de São Paulo (USP). É agente de inovação na Agência de Inovação da Universidade de São Paulo (USP).

fprado@usp.br

\section{Maria Aparecida de Souza}

É Mestre em Tecnologia Bioquímico-farmacêutica pela Universidade de São Paulo (USP). É Diretora Técnica de Propriedade Intelectual na Agência de Inovação da Universidade de São Paulo (USP).

cidin@usp.br

\section{Editor Científico: José Edson Lara}

Organização Comitê Científico

Double Blind Review pelo SEER/OJS

Recebido em 03.02.2017

Aprovado em 18.10.2017 


\title{
Resumo
}

A implementação do Núcleo de Inovação Tecnológica (NIT) ainda é apontada como um desafio no Brasil. Assim, este trabalho tem como objetivo analisar o processo de implantação e estruturação do NIT no Instituto Pasteur (IP), indicando os resultados aferidos nesse processo. Para isso, inicialmente, descrevemos o ambiente de inovação existente na instituição antes do processo; em seguida, verificamos, dentre os modelos de gestão, os que mais se aplicam às características do NIT-IP para avaliar os resultados aferidos nesse processo e, por fim, apontamos os pontos positivos e negativos do modelo usado. O IP possui atividades relacionadas a prestação de serviço e pesquisa aplicada com potencial inovador. Por isso, aqui utilizamos o modelo organizacional híbrido, com atividades voltadas para prestação de serviço e Pesquisa \& Desenvolvimento. A metodologia de pesquisa baseia-se em um estudo de caso de caráter descritivo. As ações de implantação e estruturação do NIT-IP promoveram alguns resultados positivos na instituição como institucionalização, reconhecimento e sensibilização do NIT-IP frente à comunidade científica e o acompanhamento e avaliação do potencial inovador dos projetos e resultados científicos da instituição pelo NIT-IP. Devido aos resultados obtidos, esse modelo pode ser utilizado como uma referência em outros institutos com a estrutura semelhante ou não ao IP. Contudo, o NIT-IP encontra-se em um processo de amadurecimento, devendo transpor gargalos relacionados com a ausência de verba destinada aos NIT's e recursos humanos insuficientes.

Palavras-chave: NIT's; Inovação Tecnológica; Gestão de Inovação; Modelos de Inovação.

\section{Process of implementation and structure of Technological Innovation Centers}

\begin{abstract}
Technological Innovation Centers (TIC) implementation is still identified as a challenge in Brazil. Thus, the aim here was to evaluate the implementation and structuring process of Instituto Pasteur's TIC(TIC-IP), indicating the results obtained in this process. Initially, we described the innovation environment in the Institution; then we verified between the management models, those that most apply the characteristics of NIT-IP; we evaluated during the process the results obtained; finally, we indicated positive and negative aspects of the model used. The Instituto Pasteur has activities related to public service and applied research with innovative potential. Due to the both health service activity and Research \& Development (R\&D) activities was chosen hybrid organizational model to be used. The research methodology is based on a case study of a descriptive character. The implementation and structuring actions promoted by TIC-IP produced some positive results in the institution such as institutionalization and awareness across the scientific community of TIC-IP; the monitoring and evaluation of project's innovative potential and scientific results by TIC-IP. This model can be used as a reference for other institutions with similarity or dissimilarity to the IP structure. Despite these positive results obtained in this process, the TIC-IP is in the ongoing maturation process, thus TIC-IP must overcome bottlenecks related to the lack of funding and human resources.
\end{abstract}


Keywords: TIC. Technological Innovation. Innovation Management. Innovation models.

\section{Proceso de implementación y estructuración del Centro de Innovación Tecnológica}

\section{Resumen}

La implementación del Centros de Inovación Tecnológica (CIT) se identifica como un desafío en Brasil. Así, el objetivo fue evaluar el proceso de implementación y estructuración del CIT del Instituto Pasteur (CIT-IP) indicando los resultados aferidos. Para eso, inicialmente, describimos el ambiente de innovación existente en la Institución antes del processo; em seguida verificamos entre los modelos de gestión, las que más se aplican a las características del NIT-IP; evaluamos los resultados obtenidos en este processo, y por último apuntamos los puntos positivos y negativos del modelo utilizado. Debido a la actividad de servicios de salud y las actividades de Investigación con potencial innovador se eligió el modelo de organización híbrida. La metodología de investigación se basa en un estudio de caso de carácter descriptivo. Las acciones de implementación y estructuración promovidas por CIT-IP produjeron algunos resultados positivos en la institución como la institucionalización y el reconocimiento a través de la comunidad científica de CIT-IP; la evaluación del potencial innovador del proyecto y los resultados científicos de CIT-IP. Debido a los resultados positivos obtenidos este modelo puede ser utilizado como referencia para otras instituciones con similitud o disimilitud con la estructura de IP. EI CIT-IP se encuentra en el proceso de maduración, por lo que CIT-IP debe superar los obstáculos relacionados con la falta de financiación y recursos humanos.

Palabras claves: CIT; Innovación tecnológica; Gestión de la Innovación; Modelos de innovación.

\section{Introdução}

É amplamente reconhecido o papel estratégico da inovação para redução da dependência tecnológica e aumento da competitividade do Brasil frente a outros países. Para promover a inovação, seja ela de produto, processo, tecnológica, organizacional ou de negócios, é necessário ter políticas favoráveis de gestão e incentivo à inovação (Oliveira, 2005; Cruz, 2010).

Nesse caminho, a "Lei de Inovação" foi definida como uma ação política estratégica para o país e trouxe como exigência a criação dos NIT's dentro das Instituições Científicas e Tecnológicas (ICT's), favorecendo o reconhecimento da 
participação das ICT's no processo de inovação e de proteção do conhecimento (Lei no 10.973, de 02/12/2004). Essa lei federal também institucionalizou e profissionalizou os NIT's, melhorando a comunicação entre empresa e as ICT's. Além disso, prevê mecanismos de suporte à capacitação tecnológica nas empresas e estimula a formação de novas empresas (Lei oㅜ 10.973, de 02/12/2004).

Assim, logo após seu anúncio, diversas unidades da Federação se mobilizaram para criar uma legislação análoga. No caso do Governo do Estado de São Paulo, em 2008, publicaram-se a "Lei Paulista de Inovação" e decretos, que dispõem sobre medidas de incentivo à inovação tecnológica e criam os NIT's, no âmbito das ICT's, das Secretarias de Estado (Lei complementar no. 1.049, de 19/06/2008; Decreto №. 54.690, de 18/08/2009; Decreto o․ 56.569, de 22/12/2010).

Em 2016 foi sancionado pela então Presidente da República o "novo marco legal de ciência, tecnologia e inovação", (Lei nº. 13.243, de 11/01/2016), que altera nove leis atuais. Dentre os benefícios dessa nova lei estão: licitação facilitada, estabelecendo mecanismos mais ágeis para compras e contratação de obras públicas; professores em dedicação integral de centros ou universidades públicos podem trabalhar até 8 horas por semana em atividades remuneradas no setor privado; institutos e fundações vinculadas às universidades públicas ficam autorizados a participar como sócios minoritários de empresas destinadas ao desenvolvimento de produtos ou processo inovadores, como start-ups; etc. (Lei no 13.243, de 11/01/2016).

Em relação à lei anterior, esse novo marco legal atribui aos NIT's a possibilidade de adquirir "personalidade jurídica própria", além de ampliar essas competências, como desenvolver estudos de prospecção tecnológica e de inteligência competitiva no campo da propriedade intelectual, de forma a orientar as ações de inovação da ICT; desenvolver estudos e estratégias para a transferência de inovação gerada pela ICT; promover e acompanhar o relacionamento da ICT com empresas; negociar e gerir os acordos de transferência de tecnologia oriunda da ICT (Lei №. 13.243, de 11/01/2016).

Portanto, a criação dos NIT's dentro das ICT's tem o propósito de aperfeiçoar a gestão de inovação tecnológica beneficiando a cooperação entre as ICT's e empresas. Para isso, a formação de gestores responsáveis pela interlocução entre essas entidades é essencial para tornar esse processo viável, favorável e benéfico. Ademais, a disseminação do conhecimento para as empresas brasileiras e 
estrangeiras sobre a possibilidade de trabalhar com ICT's brasileiras e a atração de interesses empresariais na pesquisa científica são igualmente importantes (Fujino, Stal \& Plonski, 1999; Segatto-Mendes \& Sbragia, 2002).

A partir do momento que as relações entre ICT, governo e empresa se intensificam, estabelece-se uma relação na qual as três esferas institucionais e as atribuições tradicionais se alternam, fazendo com que alguns atores assumam o papel de outros para o incentivo da inovação. Esse padrão de vínculos em espiral forma uma tripla hélice (Leydesdorff \& Etzkowitz, 1996; Etzkowitz, 2008).

Nesse novo ambiente de inovação, as ICT's desempenham um papel fundamental para o desenvolvimento econômico, que se adiciona ao ensino e à pesquisa. Sendo assim, a criação do NIT foi essencial para conferir aos gestores das ICT's mais autonomia em todos os temas ligados à inovação. Simultaneamente, o Decreto №. 56.569, de 22/12/2010, e a Resolução SS-53 não previram a abertura de concursos públicos para suprir e estruturar uma equipe mínima, eficiente e integrada, capaz de desempenhar as atribuições do NIT. Além disso, não presumiram um orçamento para a gestão da política de inovação nas ICT's (Decreto №. 56.569, de 22/12/2010; Resolução SS-53, de 11/05/2012).

Antes da criação da lei de inovação, algumas instituições já tinham como pauta nas suas agendas a inovação e contavam com agências de transferências de tecnologia, com atribuição de auxiliar os pesquisadores a gerenciar seus pedidos de patentes.

Uma pesquisa feita em quatro universidades brasileiras que já gerenciavam suas criações e propriedade intelectual, antes do estabelecimento da Lei de Inovação, a Universidade Estadual de Campinas (UNICAMP), a Universidade Federal do Rio de Janeiro (UFRJ), a Universidade de São Paulo (USP) e a Universidade Federal do Rio Grande do Sul (UFRGS), demonstrou que a criação dessas agências de inovação era importante na mediação da relação entre empresas e a universidade, contudo o número de tecnologias licenciadas frente aos depósitos de patentes era pequeno (Amadei \& Torkomian, 2009; Castro \& Souza, 2012).

Como podemos verificar em alguns casos, a criação dos NIT's, nas ICT's, decorreu do processo de desenvolvimento e amadurecimento e, em outros casos, para atender ao disposto na lei (Torkomian, 2009). O resultado do processo de 
criação desses núcleos nas ICT's ocasionou a definição de políticas públicas e conscientes sobre a importância da proteção das tecnologias desenvolvidas. Segundo conclui o relatório do Formulário para Informações sobre a Política de Propriedade Intelectual das ICT's do Brasil (FORMICT) de 2014, preenchido por 264 instituições públicas e privadas, no que diz respeito ao estágio de implementação dos NIT's, verificou-se que 144 instituições públicas (74,2\%) informaram que já possuem o NIT implementado, 34 instituições públicas (17,5\%) informaram que estão em fase de implementação, e apenas 16 instituições públicas (8,2\%) informaram que o NIT não está implementado. Fazendo uma análise comparativa dos últimos anos, podemos perceber que, em quatro anos, a quantidade de NIT's implementados foi ampliada em mais de $50 \%$ e suas políticas de inovação aumentaram consideravelmente, contudo a implementação e a consolidação desses núcleos ainda são apontadas como desafios nesse processo (Ministério da Ciência, Tecnologia e Inovação, 2015).

Nesse contexto, a Secretaria de Estado da Saúde de São Paulo (SES-SP), em 2012, decidiu operacionalizar a criação dos Núcleos de Inovação Tecnológica (NIT) nos institutos de saúde por meio de um programa inicial de atuação acordado entre a Coordenação de Ciência, Tecnologia e Insumos Estratégicos (CCTIES) e a Fundação Instituto de Administração (FIA), o qual foi desenvolvido ao longo de três eixos estruturantes e articulados.

As principais atividades desenvolvidas no eixo 1 foram mapeamento da maturidade de cada instituto, estruturação de modelo para o conjunto dos NIT's e suporte na formalização de processos para operacionalização de cada NIT. Já no eixo 2, as principais ações foram a capacitação dos integrantes das equipes dos NIT's. Por fim, o eixo 3 contribuiu no suporte aos processos cooperativos com outros agentes, especialmente empresas (Secretaria de Estado da Saúde, 2012a).

Para o mapeamento da maturidade das ICT's analisadas, foram realizados o diagnóstico da situação atual e potencial de desenvolvimento tecnológico das instituições, a avaliação das experiências anteriores de relacionamento com empresas e outras entidades públicas ou privadas para transferência de tecnologia, e foi verificado também o grau atual de estruturação do NIT, caso existisse. O Instituto Pasteur (IP) foi identificado no primeiro grau de maturidade em gestão de processos de inovação com práticas em potencial para gerar inovação (Secretaria de Estado da Saúde, 2012b). 
O Instituto Pasteur foi criado em 1903 e é uma instituição de pequeno porte, quando comparado com as outras instituições vinculadas a SES/SPtri, possuindo 125 funcionários, dentre eles 21 pesquisadores científicos. É uma instituição de referência no estudo da raiva, tendo como missão contribuir para a Vigilância em Saúde por meio de vigilância epidemiológica, controle de risco e programas de capacitação e desenvolvimento relativos a raiva e outras encefalites virais, abrangendo atividades laboratoriais, de pesquisa e inovação, a coordenação do programa estadual de controle da raiva, a profilaxia da raiva humana e o suporte aos municípios (Instituto Pasteur, 2015). Em relação à prestação de serviços, tem como atividades principais o diagnóstico virológico e sorológico para avaliação de anticorpos antirrábicos. É um Laboratório de Referência Nacional e consorciado da Organização Pan-Americana de Saúde (OPAS) / Organização Mundial de Saúde (OMS) para estudos de cepas do vírus da raiva. Em relação à pesquisa científica, a maioria dos projetos tem grande potencial inovador e vem sendo executada na área aplicada, visando, principalmente, à solução de problemas atuais ligados à raiva, sua epidemiologia e seu controle na população animal e humana (Instituto Pasteur, 2015).

No enfoque clássico da inovação, o IP produz e distribui insumos para diagnóstico, como o anticorpo antirrábico conjugado com isotiocianato de fluoresceína (do inglês: Fluorescein Isothiocyanate). Além disso, possui práticas potenciais no diagnóstico como a simplificação da técnica de soroneutralização para detecção e dosagem de anticorpos antirrábicos, o qual torna essa técnica mais rápida e barata. Também desenvolve técnicas de biologia molecular para identificação de diferentes espécies de mamíferos. Adicionalmente, tem competência para produzir kits para diagnóstico do vírus da raiva. No foco socioeducativo, produziu um CD interativo voltado para o público infantil com orientações sobre o controle da raiva humana e animal, que foi também traduzido para espanhol (Instituto Pasteur, 2015).

Por ter como binômio prestação de serviço e pesquisa científica, esse instituto pode ser considerado um ótimo modelo para avaliar o processo de implantação e estruturação de um NIT. Após este estudo, o modelo apresentado poderá ser utilizado por NIT's que possuem missões em comum ou não com essa instituição. 
A partir do contexto exposto, o objetivo deste estudo consiste em analisar 0 processo de implantação e estruturação do NIT no Instituto Pasteur (IP).

Para que o objetivo proposto seja atingido, foram estabelecidos os seguintes objetivos específicos:

- Descrever o ambiente de inovação existente na instituição antes do processo;

- Verificar, dentre os modelos de gestão, os que mais se aplicam às características do NIT-IP;

- $\quad$ Avaliar os resultados aferidos nesse processo;

- $\quad$ Apontar aspectos positivos e negativos do modelo usado.

\section{Referencial Teórico}

De acordo com suas atividades, os NIT's podem ser divididos em três modelos de acordo com Lotufo (2009). O primeiro modelo é o NIT com função legal, que foca suas atividades em regular e formalizar atividades de inovação na ICT e é formado por advogados e especialistas em propriedade intelectual. Outro modelo é aquele com foco em processos administrativos de aprovações e encaminhamentos para concretizar as assinaturas dos convênios e contratos referentes à interação entre ICT's e empresa. O último é aquele NIT com atividades em negócios buscando viabilizar parcerias e contratos a partir dos resultados da pesquisa nas ICT's. De acordo com o relatório Fortec, os NIT's brasileiros têm o modelo híbrido de gestão da inovação, sendo que $90 \%$ dos NIT's analisados desempenham atendimento, orientação e acompanhamento dos processos relacionados à propriedade intelectual, $86 \%$ oferecem assessoria técnica e administrativa sobre transferência e comercialização de tecnologia, $82 \%$ desses núcleos organizam eventos para disseminação da cultura de proteção à propriedade intelectual, e 76\% realizam assessoria jurídica (Torkomian, 2009).

Outra forma de categorizar os NIT's é de acordo com suas missões; os que visam à obtenção de recursos financeiros por meio dos royalties, normalmente, trabalham com tecnologias que tenham potencial maior de retorno financeiro, restringindo seu trabalho a áreas de pesquisa que são, em geral, mais rentáveis. Há os que objetivam potencializar o desenvolvimento regional a partir da transferência de tecnologia, trabalhando de forma intensa cada tecnologia com potencial de gerar uma empresa; por isso, cada profissional trabalha com poucas iniciativas e identifica 
recursos financeiros e estratégicos para os empreendimentos. $O$ terceiro grupo busca maximizar os benefícios da transferência de tecnologia à sociedade, procurando incentivar o desenvolvimento regional e desempenhar atividades mais generalistas se comparado com os dois modelos (Lotufo, 2009).

A escolha de qual modelo utilizar depende de decisões políticas institucionais e da maturidade do NIT e de sua equipe. O mais comum é a utilização de um modelo híbrido, porém é necessário definir prioridades no momento do planejamento e na estruturação de programas de transferência de tecnologias (Assumpção et al., 2010). Neste modelo de gestão híbrido, o NIT tem a função de oferecer o serviço de transferência de tecnologia e apoiar as questões jurídicas, formalizando e aprovando contratos e convênios de parceria com entidades públicas e privadas, mediante cláusulas definidas e aprovadas pela assessoria jurídica, como também facilitar o desenvolvimento de negócios a partir dos resultados da pesquisa, com o intuito de captar o investimento realizado em pesquisas (Lotufo, 2009).

Outros autores consideram, na análise organizacional dos NIT's, recursos, relação hierárquica, autonomia e incentivos (Bercovitz, Feldman, Feller, \& Burton, 2001) dividindo em três formatos: formato unitário (forma $U$ ), empresa multidivisional (forma $\mathrm{M}$ ) e empresa holding (forma $\mathrm{H}$ ). No modelo forma $\mathrm{U}$, a divisão do NIT é elaborada segundo uma perspectiva funcional, e a administração é centralizada, dividida em áreas funcionais com uma ampla linha de produtos dentro de cada área. No modelo M, o NIT é organizado a partir de produtos ou região geográfica e possui mais autonomia que o primeiro modelo, ainda que tenha um centro de decisões para temas cruciais, como alocação interna de recursos. Na terceira estrutura, forma $\mathrm{H}$, as tomadas de decisões são distribuídas entre as unidades de trabalho organizacional, isto é, entre os gerentes de cada unidade de trabalho (Bercovitz, Feldman, Feller, \& Burton, 2001).

Quanto à transferência de tecnologia (TT), existem dois tipos de estruturas: um modelo denominado do berço ao túmulo (cradle-to-grave) e o de compra e venda (Buyers-and-Sellers). No primeiro tipo, a TT é realizada por um só agente, que se responsabiliza desde a comunicação da invenção ao desenvolvimento da empresa licenciada. Esse profissional se envolve em todo o processo e, assim, cria vínculos com as pessoas envolvidas, contudo a grande desvantagem é que o sucesso da 
transferência depende somente de uma pessoa, cujo rendimento e produtividade podem afetar o resultado do processo.

Já na estrutura Buyers-and-Sellers, a transferência de tecnologia é executada por dois profissionais, um que se encarrega das questões técnicas e legais, especializado em processos de propriedade intelectual, e outro que trabalha com o licenciamento, cujo perfil é mais voltado para negócios. Nesse modelo, podem ocorrer desentendimentos e dificuldades de comunicação entre os dois profissionais que podem afetar a transferência de tecnologia, bem como tornar o processo um pouco mais complexo (Magalhães Toledo, 2009).

Os NIT's brasileiros, na grande maioria, dispõem de mais de dez profissionais, com o volume grande de estagiários, gerando assim problemas de descontinuidade e de necessidade de treinamento e capacitação constante. Além disso, outro ponto relevante é que quase $21 \%$ dos funcionários têm dedicação parcial, e $38 \%$ são estagiários e bolsistas (Ministério da Ciência, Tecnologia e Inovação, 2015). Esse fato se deve, principalmente, à falta de oferecimento de vagas específicas para esse fim nas ICT's.

\section{Metodologia}

Trata-se de uma pesquisa qualitativa de caráter descritivo do tipo estudo de caso com observação participante. A pesquisa qualitativa compreende um conjunto de diferentes técnicas interpretativas, que visa à descrição e ao processamento dos dados obtidos (Andrade, 2003). A abordagem descritiva foi adotada com o objetivo de descrever e analisar as relações entre fatos e fenômenos, permitindo o aprimoramento de ideias e a descrição das características de determinado fenômeno (Gil, 2002).

O estudo de caso é um método muito utilizado em pesquisas qualitativas, desenvolvendo-se em contexto da vida real, focalizando a realidade de uma forma complexa e contextualizada (Gil, 2002). Na observação participante, o observador se coloca na posição e ao nível dos outros elementos humanos que compõem o fenômeno a ser observado (Richardson, 2007). 


\subsection{Detalhamento da pesquisa}

Este trabalho abrangeu uma etapa constituída de pesquisa bibliográfica e uma etapa na qual foi realizado um estudo de caso. $\mathrm{O}$ objetivo da pesquisa bibliográfica foi fornecer o arcabouço teórico necessário para o desenvolvimento do trabalho. Já o estudo de caso foi elaborado de modo a se atender aos objetivos estabelecidos no planejamento da pesquisa. Baseado em revisão bibliográfica e análise do IP quanto a sua história, missão, atividades e organização, foi proposto um modelo a ser implantado no Instituto Pasteur. A partir de um modelo estabelecido de gestão, planejaram-se as etapas de implantação e estruturação do NIT-IP. Por fim, foi feita a análise do objeto de estudo para ações futuras (Figura 1).

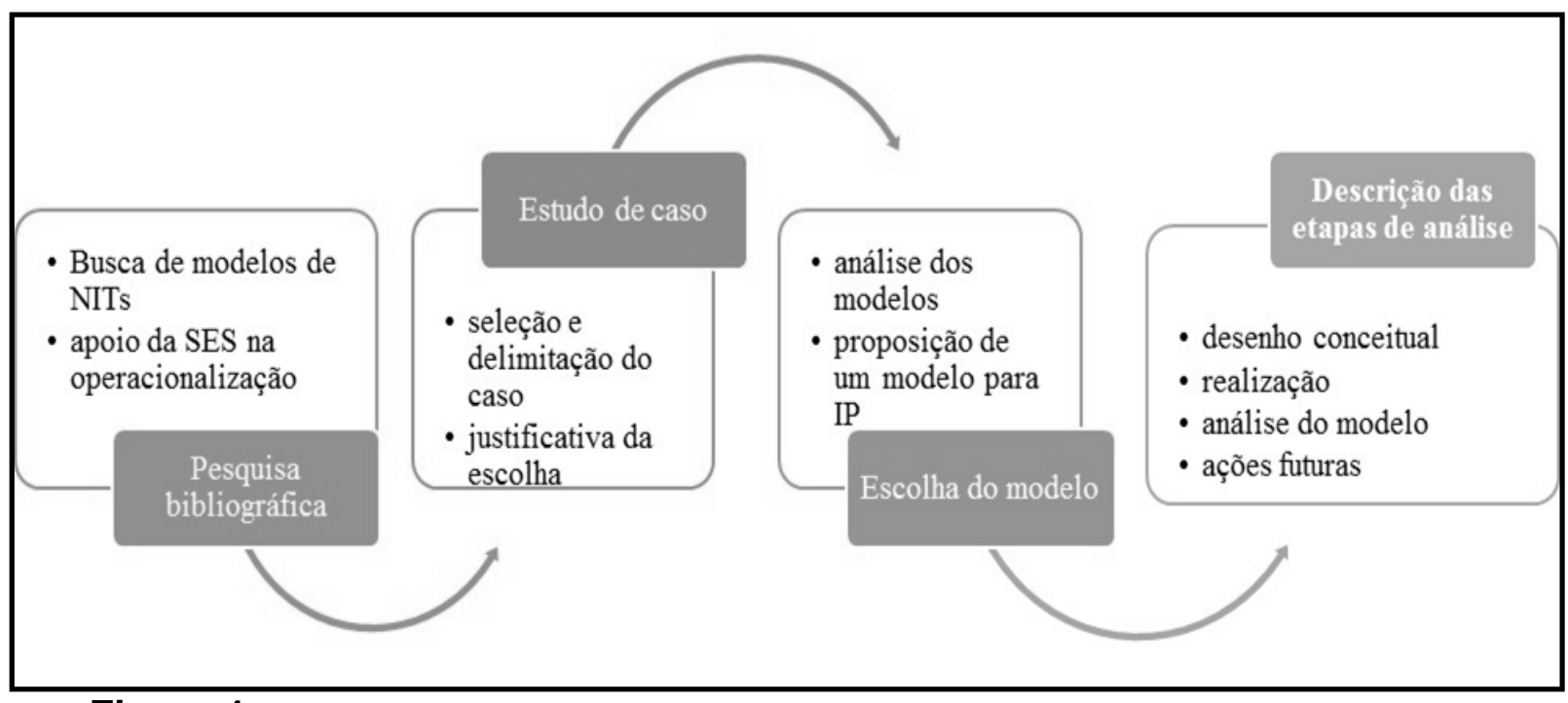

Figura 1

Etapas metodológicas

Fonte: Elaborado pelos autores. 


\subsubsection{Estudo de caso}

Para este estudo de caso, primeiramente, foi feita a seleção e delimitação do caso. Dentro desse contexto, foi selecionado o Instituto Pasteur, uma ICT de pequeno porte, com 125 funcionários, dentre esses 20 pesquisadores científicos. $O$ estudo foi delimitado no processo de implementação e estruturação do NIT do Instituto Pasteur, analisando esse processo de criação e gestão após implementação desse NIT.

A escolha do NIT-IP para a realização do estudo de caso aconteceu por vários fatores: 1) trata-se de uma ICT reconhecida, com tradição de P\&D e com demanda para a realização de depósitos de patentes; 2) trata-se de uma ICT no primeiro grau de amadurecimento quanto à gestão de inovação; 3) trata-se de uma ICT que não tinha atividades de patenteamento antes da criação do NIT; e 4) pela facilidade de acesso às informações, uma vez que o autor da pesquisa faz parte do quadro de servidores desse NIT.

Devido ao fato de o observador ser um participante deste estudo, há maiores condições de compreender hábitos, atitudes e interesses do Instituto Pasteur, quando comparado a um observador não participante. O observador, contudo, teve que tomar alguns cuidados na interpretação dos fatos.

\subsubsection{Escolha do modelo de NIT a ser implantado}

A partir das informações presentes na literatura sobre diferentes modelos de gestão de NIT's quanto a função, missão, organização e estrutura de TT, foi feita a análise e proposto um modelo de NIT que mais se adequaria ao Instituto Pasteur, de acordo com suas atividades e missões. Também foi descrito o perfil do profissional presente nessa estrutura.

\subsubsection{Descrição das etapas de análise do objeto de estudo}

Após escolha de um modelo apropriado de NIT para o IP, foi iniciada a análise das fases de implantação e estruturação do NIT, a qual foi dividida em quatro etapas sucessivas: desenho conceitual, realização, análise do modelo e ações futuras. 
$\mathrm{Na}$ fase de desenho conceitual, foi realizado o levantamento de todas as informações estratégicas para encontrar configurações que melhor se enquadrassem nos processos do Instituto Pasteur. Na etapa de realização, o grande enfoque ocorreu no aspecto funcional, para que todo o desenho realizado na fase de desenho conceitual fosse inserido no sistema de forma mais fiel possível com o planejamento inicial das atividades. Na sequência dessa fase, foi analisado o modelo utilizado após implantação e estruturação. Também foi realizado um estudo cronológico do processo de implantação e estruturação do NIT-IP. Por fim, foram planejadas as próximas ações com base na análise SWOT (do inglês: Strenghts, Weaknesses, Opportunities e Threats).

\section{Resultados e Discussão}

\subsection{Escolha do modelo de gestão do NIT-IP}

Em busca de modelos de gerenciamento dos NIT's, foi identificado o perfil dos NIT's existentes quanto a atividades, missão, organização, divisão de tarefas e recursos humanos. Essa etapa foi importante para definir o modelo a ser implantado no IP, de acordo com sua missão e atividades.

Devido ao fato de as atividades do IP serem voltadas à prestação de serviço e pesquisa aplicada, com foco em atender às demandas da sociedade, a estrutura organizacional mais adequada para o NIT-IP é o modelo de gestão híbrido. Sendo assim, o NIT-IP exercerá atividades integradas de apoio à transferência de tecnologias e negociação com diferentes organizações, como também estimular e proteger juridicamente a exploração das criações intelectuais. Dessa maneira, quanto à atividade, esse NIT deverá ter mais ênfase em negócios, auxiliando a formalização de contratos de parcerias para P\&D, e prestação de serviço, a fim de captar receita para ser investida em pesquisa, e menor ênfase em atividades ligadas a propriedade intelectual e TT com licenciamento de PI. Quando houver PI resultante de uma pesquisa, o NIT-IP poderá captar recursos na forma de royalties e outros recebimentos de PI. Nesse contexto, em virtude de o NIT-IP não ter autonomia jurídica, os contratos são encaminhados para o gestor da instituição para devidas assinaturas. 
Nesse modelo, haverá dois especialistas: um profissional que irá atuar em PI e que seja capaz de interagir com os pesquisadores, encaminhar e acompanhar as solicitações de depósitos de patentes, além de efetivar contratos com empresas que estejam interessadas no licenciamento de tecnologias de propriedade da Instituição. Já o outro especialista deverá ter como principal função negociar ativos relacionados a prestação de serviço e TT de tecnologia sem PI. Ademais, os profissionais envolvidos no NIT devem harmonizar expectativas e prestar serviços tendo os clientes e seus interesses como foco da sua atividade. Outra habilidade importante é sua capacidade de comunicação, oral e escrita, pois devem ser capazes de dar palestras, conduzir uma reunião, preparar textos técnicos e relatórios (Lotufo, 2009).

\subsection{Desenho conceitual}

Para caracterizar o estágio de cada ICT's do Estado de São Paulo no quesito da inovação tecnológica em geral e nos processos de gestão da propriedade intelectual e transferência de tecnologias, foi adotada a abordagem da Maturidade Organizacional pela SES-SP (Paulk, Weber, Garcia, Chrisssis \& Bush, 1993). Essa abordagem deriva do espraiamento de modelos de Maturidade em anos recentes.

De acordo com essa avaliação realizada pela SES-SP sobre o nível de maturidade em gestão de processos de inovação com ênfase em gestão de propriedade intelectual e transferência \& comercialização de tecnologias, o IP estava no nível 1 de maturidade, com práticas em potencial. Com tal característica, o IP foi identificado como sendo uma instituição que não tem perfil de inovação dura (geração de produtos tangíveis, tecnologicamente inovadores), contudo desenvolve serviços e processos inovadores, intensivos em conhecimento, com potencial de transferência para a sociedade.

No mapeamento tecnológico, foi identificado que o IP produz insumos para diagnóstico, que é referência para toda a Rede, com competência para produzir kits para diagnóstico. Além disso, o IP desenvolveu o teste de reservatório, no qual se identificam a biologia e o deslocamento das espécies (Carnieli Junior et al., 2008), e essa metodologia do mapeamento pode ter relevância para a inovação. No foco socioeducativo, registrou-se a produção de um CD para crianças sobre raiva, que obteve elevado sucesso e, também, foi traduzido para espanhol. 
Tendo como base o binômio prestação de serviços e pesquisa científica, o IP busca a resolução de problemas ligados à saúde pública, em particular à raiva. Pelo fato de o IP ser uma referência em raiva para o Ministério da Saúde, o conhecimento produzido tem utilidade e pode ser identificado em produtos, métodos ou processos, como também pode ser transferido na forma de conhecimento. Sua relação com as empresas se dá pelos treinamentos, prestação de serviço de diagnóstico sorológico e venda de insumos para diagnóstico de raiva.

A partir desse levantamento de dados pela direção da instituição, foi possível entender as necessidades do Instituto para conseguir implantar e estruturar o NIT de forma eficiente. Durante este estudo, foi possível identificar e planejar algumas etapas pelas quais a instituição deveria passar antes da consolidação do NIT-IP, a saber: inserção da cultura de inovação na instituição, promovendo a busca por inovações como uma prioridade estratégica; identificação e capacitação de recursos humanos para gestão do NIT; identificação e acompanhamento de projetos inovadores junto à Comissão Científica do Instituto Pasteur (CCIP), para mapeamento de anterioridade e potencial inovador de todos os projetos científicos, e levantamento de know-how do IP, assim como o levantamento de insumos estratégicos produzidos.

Outro ponto relevante no processo de implantação e estruturação do NIT-IP é que esse necessita de recursos humanos capacitados para tal finalidade. Para isso, foram identificados funcionários com o perfil desejado no modelo de gestão, e esses foram capacitados no programa específico oferecido pela SES/FIA, pelo Instituto Butantan e pela Rede Inova São Paulo.

\subsection{Realização}

\subsubsection{Recursos humanos}

A criação do Núcleo de Inovação Tecnológica (NIT) não previu a abertura de concursos públicos para suprir e estruturar uma equipe mínima, eficiente e integrada, capaz de desempenhar as atribuições do NIT, bem como não presumiu um orçamento para a gestão da política de inovação da ICT (Lei complementar no . 1.049, de 19/06/2008; Decreto №. 54.690, de 18/08/2009; Decreto №. 56.569, de 22/12/2010). Por esses motivos, a equipe técnica do NIT do Instituto Pasteur foi 
composta por seis profissionais realocados de outros setores da própria instituição para exercerem competências básicas e necessárias para realizar a gestão da inovação (Portaria IP-23, de 28/11/2013; Portaria IP-16, de 1612/2013). A escolha desses profissionais foi baseada em alguns critérios como: alto grau de qualificação técnica, conhecimento do ambiente e processos do IP e multidisciplinaridade.

Foram escolhidos dois coordenadores, ambos pesquisadores científicos com perfil inovador e empreendedor, comunicativos e com habilidade em gestão. Dentre os outros profissionais, estão dois assessores científicos com elevado mérito científico, um gestor financeiro para controle de fluxo e administração dos recursos financeiros do NIT-IP e outro profissional para realizar tarefas e rotinas administrativas.

Os coordenadores foram capacitados por meio de programas de treinamentos específicos nas várias dimensões das atividades de um NIT. A capacitação oferecida foi composta por diferentes módulos ministrados pela Fundação Instituto de Administração (FIA) e pela rede INOVA de São Paulo, como também foi constituída por um MBA de Inovação em saúde promovido pelo Instituto Butantan.

Foi também definido que, dependendo de demandas específicas, isto é, de acordo com o número de projetos identificados e selecionados, a composição do núcleo poderia ser aumentada, podendo ser agregados estudantes de Mestrado e Doutorado e de Iniciação Científica para levantamento de dados e pesquisas de campo.

\subsubsection{Atividades operacionais e estratégicas para implantação e estruturação do NIT-IP}

Após institucionalização do NIT-IP, designação da equipe e criação do regimento interno (Portaria IP-23, de 28/11/2013; Portaria IP-16, de 1612/2013), os gestores do NIT-IP caracterizaram a situação presente, do ponto de vista científico e tecnológico, econômico, estrutural e organizacional do instituto, para então identificar e priorizar ações necessárias para criação de um modelo de referência de estruturação e gestão da inovação. Os gestores do NIT-IP identificaram e elencaram as atividades necessárias para a implantação e estruturação do NIT no IP, as quais foram analisadas e colocadas como meta institucional pela direção do instituto. 
A sensibilização da comunidade técnico-científica do IP foi identificada como essencial para criar um ambiente favorável para inovação e estímulo da geração de novos conhecimentos. Foi a primeira ação, portanto, visando à estruturação do NITIP.

Para esse fim, foi ministrada uma palestra introdutória sobre a criação e atribuições do NIT-IP, cujo público-alvo era formado por pesquisadores do instituto. A sensibilização do corpo técnico foi continuada com a promoção do I Workshop do Núcleo de Inovação Tecnológica do Instituto Pasteur, cuja proposta do evento foi divulgar o Núcleo de Inovação Tecnológica (NIT) do instituto, sensibilizar os funcionários sobre a importância da inovação e estimular a reflexão sobre o papel dos institutos de pesquisa no desenvolvimento tecnológico. O evento foi estruturado da seguinte forma: apresentação de palestras, relatos de casos de sucesso, oficina prática para construção do projeto inovador e concurso para escolha do logotipo do NIT do Instituto Pasteur.

Continuando o processo de sensibilização dos funcionários do IP, foi realizada a segunda edição do Workshop de Inovação Tecnológica. Nesse evento, foi ministrada uma palestra introdutória para fomentar e incentivar o processo de inovação na instituição, como também foram apresentados casos de sucesso de empreendedores em áreas correlatas ou não com a raiva. Adicionalmente, para promover a interlocução entre os funcionários, foram promovidas atividades interativas e apresentação de resumos de trabalhos com potencial inovador do IP.

Com a finalidade de apresentar, de forma objetiva e didática, os conceitos básicos sobre os temas como Inovação, Propriedade Intelectual e Transferência de Tecnologia, foi publicada a primeira edição da Cartilha de Propriedade Intelectual e Inovação do NIT-IP (Katz, 2015).

Essas ações de sensibilização tiveram como resultado o aumento da busca do NIT-IP pelo inventor, como também o aumento do número de projetos com potencial inovador cadastrados na instituição.

Visando ser proativo e contribuir na resolução dos problemas prioritários de saúde da população e para o fortalecimento da gestão do Sistema Único de Saúde, - NIT-IP participou das duas últimas Oficinas de Seleção de Prioridades do Programa Pesquisa para o SUS (PPSUS). Nessas oficinas, os coordenadores do 
NIT-IP, bem como pesquisadores e gestores de outros NIT's, definiram os eixos e as linhas de pesquisas prioritárias para saúde.

Para a identificação de boas práticas de gestão da inovação de outros NIT's em fase de implementação, implementados ou consolidados que podem colaborar no desenvolvimento do NIT-IP, a ação estratégica utilizada foi a articulação com esses NIT's por meio de redes. Nesse sentido, podemos destacar a Rede de NITSES formada com o subsídio da CCTIES, da participação da Rede Inova São Paulo o NIT-IP, bem como apoiando outros eventos para fortalecimento das redes como o $8^{\circ}$ Fórum Nacional de Gestores de Inovação e Transferência de Tecnologia (Fortec).

Outra conduta que foi identificada como fundamental nesse processo foi a gestão estratégica de projetos de P\&D do IP. Para isso, o NIT-IP auxilia a Comissão Científica do IP na avaliação, acompanhamento e monitoramento de atividades de pesquisa que tenham potencial inovador e que sejam realizadas na instituição ou em parceria com outras instituições. Nesses projetos, são avaliados os níveis de complexidade e demais fatores de riscos e incerteza baseados no modelo Diamante (Shenhar \& Dvir, 2007). Esse modelo se baseia em quatro dimensões dos projetos: inovação (incertezas nos objetivos); tecnologia (incertezas tecnológicas); complexidade (mede a complexidade do produto, tarefa e organização); ritmo ou passo (grau de urgência ou prioridade pelo cliente ou mercado) (Shenhar \& Dvir, 2007).

Também foram avaliadas a alocação e a carga de trabalho dos recursos financeiros e humanos usados nesses projetos. As tecnologias identificadas como inovadoras são divulgadas no portfólio criado na SES-SP. Junto à CCIP, foi realizado um mapeamento de anterioridade e potencial inovador de todos os projetos científicos terminados ou em andamento, assim como um levantamento de know-how do IP e de insumos estratégicos produzidos.

Abaixo segue a cronologia das etapas de implantação e estruturação do NITIP (Figura 2). 


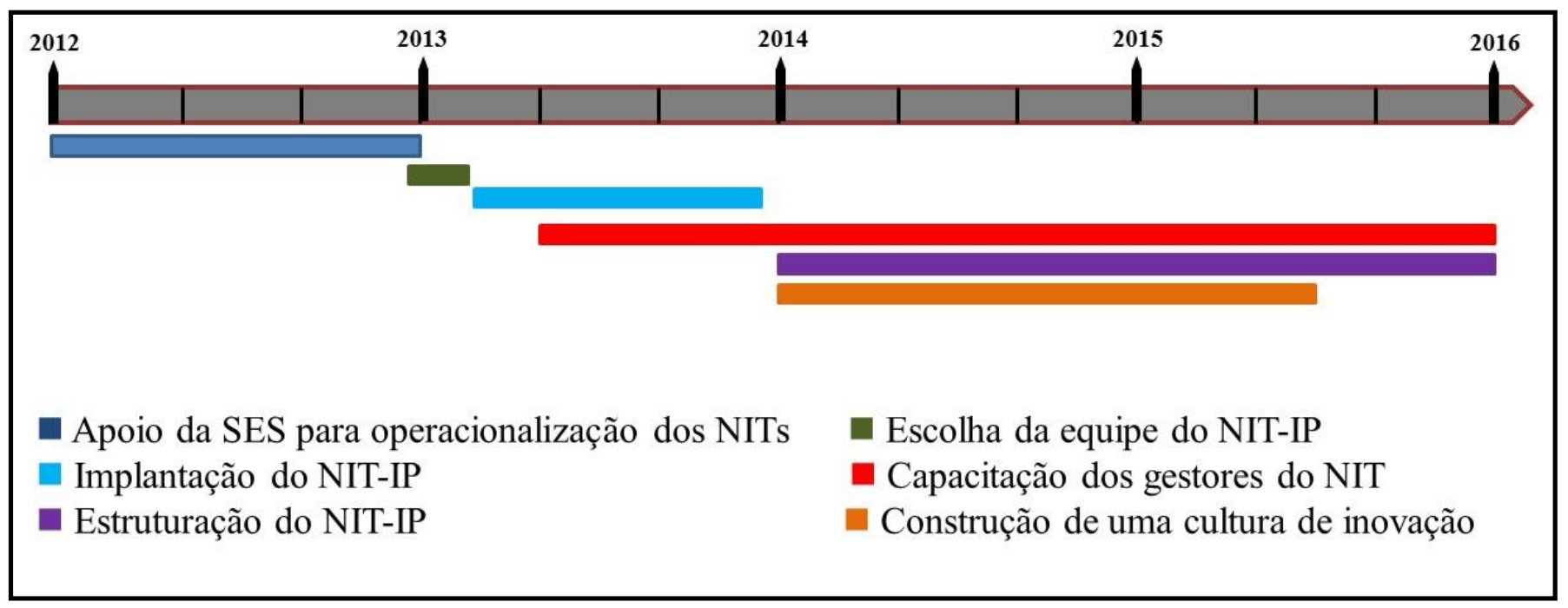

Figura 2

Ordem cronológica das etapas de implantação e estruturação do NIT-IP.

Fonte: elaborada pelos autores.

\subsection{Análise do modelo de gestão}

A escolha por funcionários da instituição para gerir o NIT-IP foi positiva nos seguintes aspectos: os coordenadores do NIT, por serem pesquisadores científicos e capacitados quanto ao processo de inovação, têm expertise em ciência, inovação, propriedade intelectual, legislação e negócios, facilitando a articulação e gestão dessas diferentes áreas no modelo de gestão híbrido escolhido; ausência de custos com a contratação de profissionais para administrar o NIT-IP; a equipe possui conhecimento nos processos administrativos e técnicos do IP. Esses funcionários, todavia, não atuam em regime de dedicação exclusiva em relação às atribuições do NIT, estando envolvidos com outras atividades dentro da instituição.

Observou-se que a realização dos eventos de Inovação Tecnológica promovidos pelo NIT-IP sensibilizou os funcionários da instituição com relação ao tema, com a maioria dos participantes pertencentes às distintas áreas. Além disso, as palestras proferidas permitiram a compreensão de diferentes temas relacionados à inovação tecnológica, promovendo maior conhecimento sobre o assunto. Observou-se, também, que essa iniciativa motivou os pesquisadores a buscar os serviços do NIT do IP e aumentou o número de projetos com potencial inovador cadastrados na Comissão Científica do Instituto Pasteur. Como mostrado 
anteriormente, o êxito dessas ações se deve, em grande parte, ao apoio da direção do Instituto no processo de implantação e estruturação do NIT-IP, contudo, para manter o bom desempenho na tarefa de sensibilização contínua da comunidade científica, o NIT necessita estar respaldado pela política institucional, que deve contemplar a preocupação com a proteção da produção intelectual, antes de serem divulgados os resultados de pesquisa.

A identificação, a análise e o acompanhamento de projetos com potencial inovador do IP permitiram que o NIT-IP classificasse os resultados decorrentes de projetos de pesquisa, opinasse quanto à proteção ou divulgação das criações desenvolvidas na instituição, além de assegurar que as medidas de proteção legal e sigilo da P\&D sejam tomadas em concordância com os objetivos do instituto.

Analisando a ordem cronológica das etapas do processo de implantação e estruturação do NIT-IP e o tempo despendido para cada fase, foi verificado que: (a) houve uma ação de curto prazo, com duração de um ano, que envolveu o projeto de apoio da SES para a implantação do NIT-IP, que precedeu à criação desse NIT e foi essencial neste projeto; (b) as ações que se enquadram como de médio prazo foram a escolha da equipe gestora desse NIT e capacitação da equipe para dar continuidade a esse processo; (c) as ações de médio/longo prazo foram a estruturação do NIT e a promoção de uma cultura de inovação no instituto. Observou-se, também, que a capacitação da equipe do NIT, que é uma ferramenta de motivação dos funcionários, deve ser contínua e que a sensibilização da equipe sobre a importância da inovação é uma ação que demanda tempo e é extremamente favorável para guiar o trabalho dos pesquisadores, além de auxiliar a aderência da comunidade interna ao plano de ações da instituição e a sua concretização (Figura 2).

A Análise SWOT é uma ferramenta utilizada para fazer análise de cenário (ou análise de ambiente), sendo usada como base para gestão e planejamento estratégico de uma instituição ou empresa, podendo ser utilizada para qualquer tipo de análise de cenário. O termo SWOT é uma sigla oriunda da língua inglesa, um acrônimo para pontos fortes (strenghts), pontos fracos (weakenesses), oportunidades (opportunities) e ameaças (threats), criada por Andrewns e Roland Christensen (Hindle \& Lawrence, 1994). A análise SWOT do processo de implantação e estruturação do NIT-IP está apresentada abaixo e foi utilizada para conduzir e planejar as próximas atividades do NIT-IP (Figura 3). 


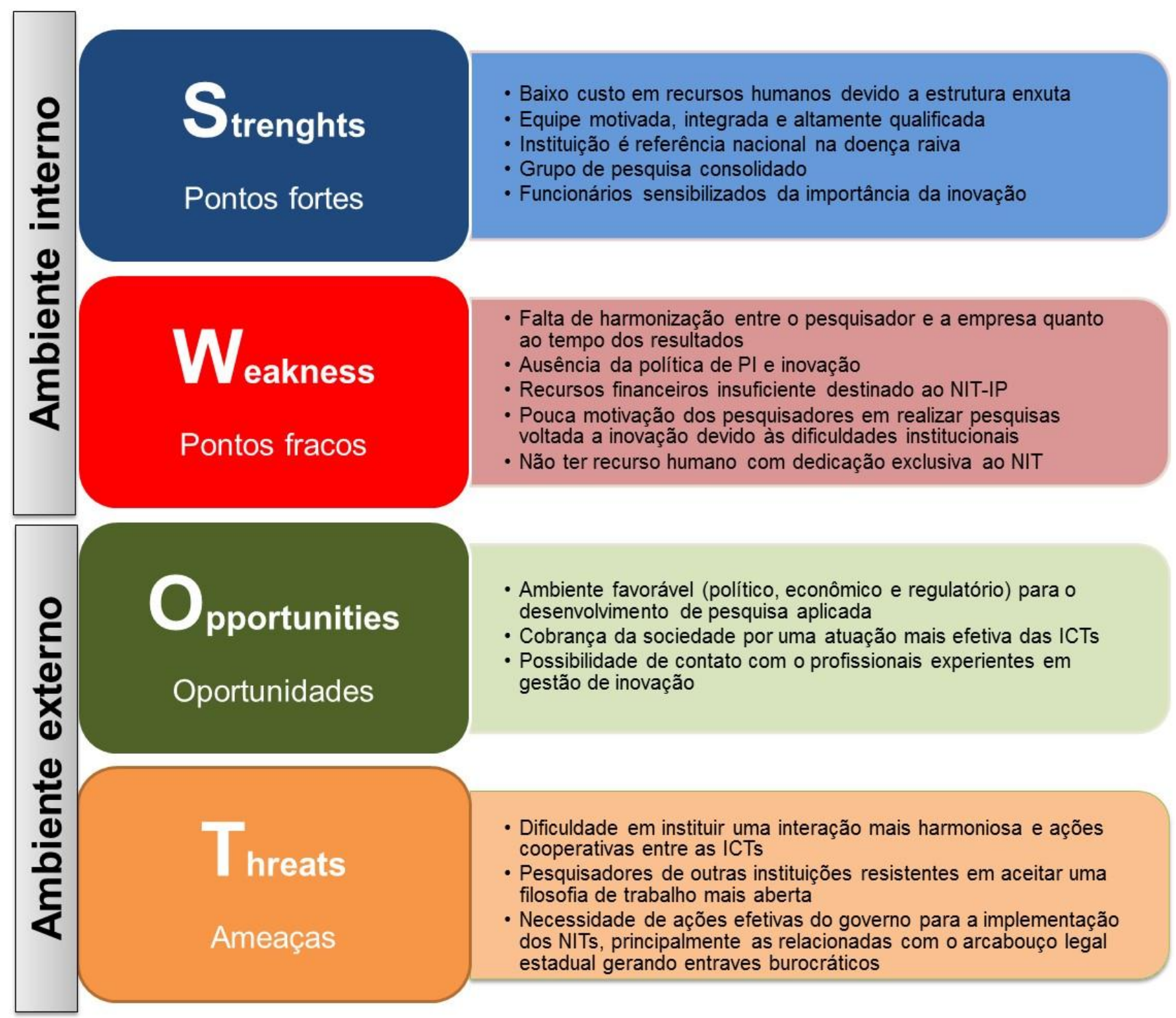

Figura 3

Análise com a ferramenta SWOT do NIT-IP

Fonte: Elaborado pelos autores.

\subsection{Planejamento das próximas ações para a consolidação do NIT-IP}

Após a análise do modelo implementado, constatou-se que, para o desenvolvimento do NIT, no Instituto Pasteur, é necessária a adoção de ações que sigam os princípios de qualidade, flexibilidade e agilidade nos serviços prestados. Além disso, como já apontado, o NIT-IP deve estar alinhado com a missão do instituto. Dessa forma, o modelo de gestão híbrido escolhido é o mais adequado, pois visa harmonizar as atividades de negócios, como prestação de serviço para 
captar receita, como também oferecer serviço de Transferência de Tecnologia (TT) auxiliando a formalização de contratos de parcerias para P\&D.

Nesse contexto, o planejamento estratégico para futuras ações incluiu elementos listados apontados como fraquezas e ameaças na análise SWOT. Dentre elas, podemos destacar a manutenção e a consolidação das atividades atuais, como a sensibilização continuada por meio de realização de palestras e seminários, visando ao amadurecimento dos profissionais quanto à importância de formas de proteção intelectual, seus benefícios e implicações, assim como da motivação para o desenvolvimento profissional, para o trabalho criativo e coletivo e para a atitude empreendedora.

Outra atividade que deverá ser continuada será a participação e colaboração na Rede de NIT da SES/SP, na Rede Inova São Paulo e em eventos como o Fortec. Essa ação visa aumentar a interação do NIT-IP com outros NIT's, proporcionando, assim, ações colaborativas entre os NIT's, como também a sensibilização coletiva do corpo técnico-científico.

Outro ponto fraco apontado foi a ausência de uma política de inovação na instituição, importante para a organização e a gestão dos processos que orientam a transferência de tecnologia e a geração de inovação no Instituto Pasteur. Sendo assim, a elaboração da Política de Inovação institucional para regulamentar as ações do NIT com políticas explícitas para a consultoria e a prestação de assistência técnica ou científica será outra medida para consolidação do NIT-IP. Nesse contexto, também padronizaremos os manuais de procedimentos e formulários específicos (relatórios de invenção, modelos de acordos, contratos ou convênios, cartas de encaminhamento), uniformizando as atividades deste NIT.

Para aprimorar a análise dos projetos de P\&D com potencial inovador e garantir a proteção das invenções, faremos análises quanto a viabilidade econômica e retorno social para uso adequado dos recursos disponíveis no IP.

Com menor foco em PI, o NIT-IP estabelecerá um fluxo de acompanhamento do depósito, tramitação, vigilância, pagamentos de anuidades da PI e gestão do portfólio de patentes do Instituto Pasteur para proteger esse conhecimento adequadamente. Contudo, devido à falta de recursos humanos especializados em direito em PI, como apontado na análise SWOT, o serviço de consultoria, assessoria e gestão dos direitos da Propriedade Intelectual, como também o auxílio na busca 
de anterioridades e na redação de patentes, será terceirizado por empresa especializada nesses procedimentos.

Com o foco principal na viabilização de parcerias, o NIT-IP irá realizar prospecção, mapeamento e identificação de potenciais parceiros de instituições públicas ou privadas, estabelecer os contatos preliminares e as providências para as primeiras reuniões de discussão sobre o tema específico e a possível formalização de parcerias visando à transferência de tecnologias e prestação de serviço.

Por fim, a criação de indicadores para mensuração dos resultados e de gestão do desempenho do NIT-IP também será uma atividade estratégica para revisar e aprimorar os processos de gestão da inovação desse núcleo.

\section{Considerações Finais}

A partir da promulgação da Lei da Inovação Federal e Estadual (Lei ํㅜ. 10.973, de 02/12/2004; Lei complementar ํ․ 1.049, de 19/06/2008), institucionalizou-se a criação dos NIT's, cuja principal atribuição é a gestão da produção de inovações em suas instituições. É escassa, contudo, a literatura sobre como conduzir a implantação e estruturação de NIT's de ICT's.

Nesse aspecto, o presente artigo procurou analisar o processo de implantação e estruturação do NIT no Instituto Pasteur por ser uma ICT que tem missão híbrida, com prestação de serviço, pesquisa científica, e também a necessidade de atender a uma demanda da sociedade.

Assim, após caracterizar o ambiente de inovação existente no IP antes do processo de implantação e estruturação e identificar alguns profissionais na instituição com perfil para gerir o NIT-IP, foi determinado o modelo de gestão a ser implementado. Esse modelo foi escolhido com base na missão e nas atividades do Instituto Pasteur, bem como na maturidade e na formação da equipe do NIT.

Também foram planejadas ações estratégicas para que esse processo tivesse êxito. Dentre essas, foram elencadas como principais medidas a sensibilização da comunidade técnico-científica e a gestão estratégica de projetos de P\&D. Nesse sentido, essas atividades promoveram a sensibilização e o interesse da comunidade científica com o tema, assegurando o acompanhamento pelo NIT-IP de projetos com potencial inovador. A participação do NIT-IP na Rede de NIT's da SES/SP e na Rede 
Inova São Paulo foi essencial para identificação e padronização de boas práticas de gestão da inovação de outros NIT's já consolidados.

No contexto do NIT-IP, foi observado que fraquezas e ameaças relacionadas com timing do pesquisador e da empresa quanto ao tempo e resultados de pesquisa esperado e a motivação do pesquisador, mesmo com as dificuldades institucionais, podem ser solucionadas ou minimizadas com programas de sensibilização contínua do corpo técnico. Já a falta de recursos humanos com dedicação exclusiva e a escassa verba destinada ao NIT dependem de ações governamentais. Contudo o NIT-IP possui um cenário interno favorável para sua consolidação, com uma equipe eficiente e de baixo custo, como também o cenário econômico e político favorável ao desenvolvimento de projetos inovadores.

Também foi observado o aumento crescente da procura do NIT-IP pelos inventores da instituição, bem como o aumento do número de projetos com potencial inovador cadastrados na Comissão Científica. Esses dados são bons indicadores do sucesso desse processo.

O NIT-IP, na fase de consolidação desse núcleo, terá a função de promoção da articulação entre o IP e entidades públicas ou privadas, com o objetivo de captar parcerias e financiamentos junto a esses órgãos. Nesse sentido, o avanço na experiência desse NIT pode evoluir de uma simples negociação entre empresa e instituição pública para uma aliança estratégica entre as ICT's. Após o NIT bem estabelecido, é viável um exercício constante de SWOT para identificar as forças e as fraquezas dele e das prováveis oportunidades e ameaças relacionadas com sua atuação.

Apesar deste trabalho apresentar, passo a passo, o processo de implantação e estruturação do NIT, podendo contribuir para direcionar a criação de outros NIT's que tenham o mesmo escopo desee NIT, ou mesmo NIT's com características distintas, as estratégias escolhidas não podem ser assumidas como um modelo rígido e padronizado. O outro fator importante é que os métodos devem ser modificados conforme a interação ou a competência dos atores envolvidos no processo de inovação dentro da instituição. 


\section{Referências}

Amadei, J. R. P., \& Torkomian, A. L. V. (2009). As patentes nas universidades: análise dos depósitos das universidades públicas paulistas (1995-2006). Ciência da Informação, 38 (2), 9-18.

Andrade, M. M. (2003). Introdução à Metodologia do Trabalho Científico: elaboração de trabalhos na graduação (6a ed.). São Paulo: Atlas.

Assumpção, F. C., Pereira, C. L., Mascarenhas, I. M., Rugani, I. G., Pirola, J. L., \& Varneiro, M. S. (2010). Estruturação e Planejamento de Núcleos de Inovação Tecnológica. Florianópolis: PRONIT- Implantação e estruturação do arranjo catarinense de núcleos de inovação.

Bercovitz, J., Feldman, M., Feller, I., \& Burton, R. (2001). Organizational Structure as a Determinant of Academic Patent and Licensing Behaviour: An exploratory study of Duke, Johns Hopkins, and Pennsylvania State Universities. Journal of Technology Transfer, 26, 21-35.

Carnieli Junior, P., Fahl, W. de O., Castilho, J. G., Brandão, P. E., Carrieri, M. L., \& Kotait, I. (2008). Species determination of Brazilian mammals implicated in the epidemiology of rabies based on the control region of mitochondrial DNA. The Brazilian Journal of Infectious Diseases, 12(6), 462-5.

Castro B. S., \& Souza G. C. (2012). O papel dos Núcleos de Inovação Tecnológica (NIT's) nas universidades brasileiras. LIINC em Revista, 8(21), 125-140.

Cruz, C. H. B. (2010). Ciência, tecnologia e inovação no Brasil: desafios para o período 2011 a 2015. Interesse Nacional, ano 3, (10).

Decreto no 54.690 (2009, 18 de agosto). Diário Oficial do Estado de São Paulo Seção 1, p. 1. São Paulo, SP.

Decreto no 56.569 (2010, 22 de dezembro). Diário Oficial do Estado de São Paulo, Seção 1, p. 4. São Paulo, SP.

Etzkowitz, H. (2008). The Triple Helix - University-Industry-Government Innovation in Action. New York: Routledge, 164.

Fujino, A., Stal, E., \& Plonski, G. A. (1999). A proteção do conhecimento na universidade. Revista de administração, 34(4), 46-55.

Gil, A. (2002). Como elaborar projetos de pesquisa. (4a ed.). São Paulo: Atlas.

Hindle, T., Lawrence, M. (1994). Field Guide to Strategy: A Glossary of Essencial Tools and Concepts of Today Managers. Harvard Business School Press.

Instituto Pasteur. Disponível em: <http:// www.pasteur.saude.sp.gov.br /> 
Katz, I. S. S. (2015). Cartilha de Propriedade Intelectual e Inovação (1a ed.) São Paulo: Núcleo de Inovação Tecnológico (NIT) do Instituto Pasteur.

Lei complementar n. 1.049, de 19 de junho de 2008. (2008, 19 de junho). Diário Oficial Estadual, Seção 1, p. 114. São Paulo, SP.

Lei n. 10.973, de 02 de dezembro de 2004. (2004, 22 de dezembro). Diário Oficial da União Seção 1, p. 2, Brasil.

Lei $\mathrm{n}^{\circ}$ 13.243, de 11 de janeiro de 2016. (2016, 11 de janeiro). Diário Oficial da União Seção 1, p. 1, Brasil.

Leydesdorff, L., \& Etzkowitz, H. (1996). The future location of research: a triple helix of university-industry-government relations II. Easst Review, 15(4), 20-25.

Lotufo, R. A. (2009). A institucionalização de Núcleos de Inovação Tecnológica e a experiência da Inova Unicamp. In Santos, M. E. R. (Org.), Toledo, P. T. M., \& Lotufo, R. A. Transferência de Tecnologia - Estratégias para a estruturação e gestão de Núcleos de Inovação Tecnológica. Campinas: Komedi, 41-73.

Magalhães Toledo, P. T. (2009). A Gestão Estratégica de Núcleos de Inovação Tecnológica: Cenários, Desafios e Perspectivas. In Seminário LatinoIberoamericano de Gestión Tecnológica (Altec 2009), Cartagenas de Indias.

Ministério da Ciência, Tecnologia e Inovação (2015). Política de Propriedade Intelectual das Instituições Científicas e Tecnológicas do Brasil - Relatório Formict 2014. Recuperado de http://www.mct.gov.br/upd blob/0237/237597.pdf

Oliveira, D. H. (2005). O futuro da indústria: cadeias produtivas. Brasília, 1, 11-38.

Paulk, M. C., Weber, C. V., Garcia S. M., Chrissis, M. B., \& Bush M. Key. (1993). Practices of the Capability Maturity ModelSM, Version 1.1.1 Ed. Technical Report. Pittsburgh, Pennsylvania: Software Engineering Institute, Carnegie Mellon University.

Portaria IP-16, de 16/12/2013, São Paulo (Estado). Diário Oficial do Estado de São Paulo, Poder Executivo Seção I, p.32. São Paulo, SP.

Portaria IP-23, de 28/11/2013, São Paulo (Estado). Diário Oficial do Estado de São Paulo, Poder Executivo Seção I. São Paulo, SP.

Resolução SS-53 de 10/05/2012. Diário Oficial do Estado de São Paulo, Seção 1, p. 35. São Paulo, SP.

Richardson, R. J. (2007). Pesquisa social: métodos e técnicas. (3a ed.). São Paulo: Atlas.

Secretaria de Estado da Saúde. (2012a). Serviços técnicos especializados para implementação dos núcleos de inovação tecnológica nos institutos que operam no 
âmbito da SES, Relatório 1, São Paulo. Recuperado de: http://ses.sp.bvs.br/wpcontent/uploads/2014/09/relat\%C3\%B3rio-parcial-1 evolu\%C3\%A7\%C3\%A3oproj.NIT'S .pdf.

Secretaria de Estado da Saúde. (2012b). Serviços técnicos especializados para implementação dos núcleos de inovação tecnológica nos institutos que operam no âmbito da SES, Relatório 2, São Paulo. Recuperado de: http://ses.sp.bvs.br/wpcontent/uploads/2014/09/relat\%C3\%B3rio-parcial-2 evolu\%C3\%A7\%C3\%A3oproj.-NIT'S.pdf.

Segatto-Mendes, A. P., Sbragia, R. (2002). O processo de cooperação universidadeempresa em universidades brasileiras. Revista de Administração. 37(4), 58-71.

Shenhar, A. J., \& Dvir, D. (2007). Reinventing project management: the diamond approach to successful growth and innovation. Boston: Harvard Business School.

Torkomian, A. L. V. (2009). Panorama Dos Núcleos De Inovação Tecnológica No Brasil. In Santos, M. E. R. dos, Toledo, P. T. M. de, \& Lotufo, R. de A. Transferência de Tecnologia: estratégias para estruturação e gestão dos Núcleos de Inovação Tecnológica. Campinas: Komedi, 21-38. 